\title{
Ubiquity of non-geometry in heterotic compactifications
}

\author{
Iñaki García-Etxebarria, ${ }^{a}$ Dieter Lüst, ${ }^{a, b}$ Stefano Massai ${ }^{c, b}$ and Christoph Mayrhofer ${ }^{b}$ \\ ${ }^{a}$ Max Planck Institute for Physics, \\ Föhringer Ring 6, 80805 Munich, Germany \\ ${ }^{b}$ Arnold Sommerfeld Center for Theoretical Physics, \\ Theresienstraße 37, 80333 Munich, Germany \\ ${ }^{c}$ Enrico Fermi Institute, University of Chicago, \\ 5640 S Ellis Ave, Chicago, IL 60637, U.S.A. \\ E-mail: inaki@mpp.mpg.de, dieter.luest@lmu.de, massai@uchicago.edu, \\ christoph.mayrhofer@lmu.de
}

ABSTRACT: We study the effect of quantum corrections on heterotic compactifications on elliptic fibrations away from the stable degeneration limit, elaborating on a recent observation by Malmendier and Morrison. We show that already for the simplest nontrivial elliptic fibration the effect is quite dramatic: the $I_{1}$ degeneration with trivial gauge background dynamically splits into two T-fects with monodromy around each T-fect being (conjugate to) T-duality along one of the legs of the $T^{2}$. This implies that almost every elliptic heterotic compactification becomes a non-geometric T-fold away from the stable degeneration limit. We also point out a subtlety due to this non-geometric splitting at finite fiber size. It arises when determining, via heterotic/F-theory duality, the SCFTs associated to a small number of pointlike instantons probing heterotic ADE singularities. Along the way we resolve various puzzles in the literature.

Keywords: F-Theory, String Duality, Superstrings and Heterotic Strings, Superstring Vacua

ARXIV EPRINT: 1611.10291 


\section{Contents}

1 Introduction 1

2 Heterotic/F-theory duality map 2

3 Quantum splitting of the $I_{1}$ degeneration in the $E_{8} \times E_{8}$ heterotic string 4

3.1 One NS5 on top of the $I_{1}$ degeneration 5

3.2 Moving the NS5 away from the degeneration point 6

$\begin{array}{ll}3.3 \text { Genericity of the splitting } & 10\end{array}$

4 F-theory interpretation of the $\mathrm{SO}(32)$ splitting $\quad 11$

$\begin{array}{ll}4.1 \text { The stable degeneration limit } & 13\end{array}$

$\begin{array}{lll}4.2 & \text { Dualizing the } I_{1} \text { degeneration } & 14\end{array}$

5 Relation to previous work $\quad \mathbf{1 5}$

5.1 Genus-two formulation of $E_{8} \times E_{7}$ compactifications $\quad 15$

5.2 Absence of bare $I_{m}$ singularities in the genus two formalism 16

$\begin{array}{ll}5.3 \text { Non-geometric unfreezing of moduli spaces } & 16\end{array}$

$\begin{array}{ll}5.4 \text { A subtlety at low instanton number } & 17\end{array}$

6 Discussion 18

\section{Introduction}

In this note we study the behavior of the heterotic string on an elliptically fibered K3, in the regime where the ten-dimensional gauge group stays unbroken. In other words, the full non-triviality of the gauge bundle is concentrated at points on the K3, where NS5 branes are located [1]. While this is well-trodden territory, we will show that one important aspect of the quantum dynamics of this background has been overlooked.

The result is simplest to state, and most striking, in the case of the local background given by the $I_{1}$ degeneration of the elliptic fibration, in the absence of neighboring NS5 branes, i.e. a purely geometric background. The total space of the elliptic fibration is in this case smooth. We will take the size of the fiber to be finite. We show that in this very simple background, quantum effects lead to a splitting of the $I_{1}$ degeneration into a pair of non-geometric T-fects - using nomenclature borrowed from [2, 3]. Around each of these defects of the background, the monodromy on the $T^{2}$ fiber of the fibration is (conjugate to) T-duality along one of the legs of the $T^{2}$. This implies that almost every heterotic background is described by a T-fold [4], once we take the quantum dynamics into account. The relevant quantum dynamics is in fact not exotic: in the case of the $\mathrm{SO}(32)$ heterotic string the relevant effects are worldsheet-instantons wrapping the $T^{2}$ fiber, which dualize 
to the well-known $\mathrm{D}(-1)$-instanton effects in F-theory that split the $\mathrm{O}^{-}$plane into its $B$ and $C$ components [5-7].

The physical reason for why the splitting happens is analogous to the reason for the familiar $\mathrm{O}^{-}$splitting: in the heterotic string we have a modified Bianchi identity that gives rise to a "wrong sign" monodromy for the integral of the $B_{2}$-field over the $T^{2}$ fiber as we go around an $I_{1}$ degeneration. We have that, encircling $I_{1}$ counterclockwise, $b$ goes to $b-1$, in conventions where $b$ is the real part of the complexified Kähler modulus $\rho=\int_{T^{2}} B_{2}+i \operatorname{Vol}\left(T^{2}\right)$ and the monodromy around an NS5 is $b \rightarrow b+1$. In order to obtain such a monodromy from the naïve solution to the equation of motion for $\rho$ would force us to set the volume of the $T^{2}$ fiber to negative values. ${ }^{1}$ The heterotic string cures this sickness of the innocent-looking $I_{1}$ degeneration by becoming a T-fold. In other words, the "bare" heterotic $I_{1}$ splits into two T-fects with the combined monodromy around the two T-fects being the desired $b \rightarrow b-1$, but each T-fect having a more involved monodromy.

This viewpoint also suggests that once we put one or more NS5 branes on top of the $I_{1}$ degeneration of the fibration there should be no quantum splitting anymore, and one should recover the geometric interpretation. This is indeed the case, as we will show.

As in [3, 9-11], our approach to understand the non-geometry of the heterotic string will be to use the map to F-theory [12]. For our purposes it will suffice to understand the duality map in the case of unbroken gauge symmetry, ${ }^{2}$ which was worked out in the stable degeneration limit in $[14,15]$, and away from the stable degeneration limit (the case of most interest to us) in [16] (see also [9, 17, 18]). In section 3 we will use this map for the simplest system of an $I_{1}$ degeneration of an elliptic fibration over flat space, with and without an NS5 brane. We will show that generically the $I_{1}$ degeneration splits, and we will analyze the nature of its T-fect constituents. In section 4 we analyze the case of the $\mathrm{SO}(32)$ heterotic string, and relate the quantum splitting in this case to the well known splitting of the $\mathrm{O}^{-}$plane in the dual F-theory description. In section 5 we explain how our observation sheds light on various obscure points in the literature. We finish in section 6 by listing some interesting open questions.

\section{Heterotic/F-theory duality map}

For simplicity, during most of our analysis we take a noncompact base for the elliptic fibration, so we have an elliptic fibration over $\mathbb{C}$, which we parameterize by a complex coordinate $s$. We will denote the coordinates on the elliptic fiber, given in Weierstraß form, by $[x: y: z]$. The dual F-theory background is given by a K3 fibration over our $\mathbb{C}$ base, with the K3 itself elliptically fibered. We denote by $[u: v]$ the coordinates on the $\mathbb{P}^{1}$ base of the K3, and reuse $[x: y: z]$ for the coordinates of the $\mathbb{P}^{2,3,1}$ in which the elliptic fiber is embedded. It should be always clear from the context which $T^{2}$ we are discussing.

\footnotetext{
${ }^{1}$ The argument here is analogous to the one leading to the naïve negative IIB string coupling $g_{s}$ close to an $\mathrm{O}^{-}$. For a review of the details see for instance [8] and references therein.

${ }^{2}$ There has been recent interesting progress in understanding the $E_{7} \times E_{8}$ case $[10,11,13]$, and in fact our discussion sharpens the observation in [10] that the moduli space of the non-geometric heterotic $E_{7} \times E_{8}$ on K3 agrees with the full moduli space of the family of F-theory geometries dual to certain geometric heterotic compactifications, as we discuss in more detail in section 5 .
} 
In this section we will focus on the case of the $E_{8} \times E_{8}$ heterotic string, leaving the analysis of the $\mathrm{SO}(32)$ heterotic to section 4.

For the convenience of the reader, let us first review the eight dimensional map explicitly constructed in [16-18]. Start with an elliptically fibered K3 (giving the fibration of the F-theory background) of the form

$$
y^{2}=x^{3}+a u^{4} v^{4} x z^{4}+\left(b u^{5} v^{7}+c u^{6} v^{6}+d u^{7} v^{5}\right) z^{6} .
$$

Parameterizing the complex structure and complexified Kähler modulus of the dual heterotic $T^{2}$ by $\tau$ and $\rho=\int_{T^{2}}(B+i J)$, respectively, we have

$$
\begin{aligned}
j(\tau) j(\rho) & =-12^{6} \frac{a^{3}}{27 b d}, \\
\lambda(\tau) \lambda(\rho) & =12^{6} \frac{c^{2}}{4 b d}
\end{aligned}
$$

where we have introduced $\lambda=12^{3}-j$. In the holomorphic setting ${ }^{3} \tau$ and $\rho$ are only defined up to the duality action $\left(\mathrm{SL}(2, \mathbb{Z})_{\rho} \times \mathrm{SL}(2, \mathbb{Z})_{\tau}\right) \rtimes \mathbb{Z}_{2}$. Hence a map such as (2.2), given by a symmetric function on the $j$-functions, is the best that we can hope for.

Before proceeding, let us briefly recall some useful basic facts about elliptic curves. It will be useful below to introduce $\kappa=j / \lambda$. Given a particular $j$ invariant for an elliptic curve, one can construct an elliptic curve with the same invariant by taking the hypersurface

$$
y^{2}=x^{3}+3 \kappa x z^{4}+2 \kappa z^{6}
$$

in $\mathbb{P}^{2,3,1}$, as one can easily check. For any choice of the coefficients $f$ and $g$, appearing in the Weierstraß representation, of the elliptic curve with a given $j$-invariant, one has that

$$
\lambda=12^{3}-j=12^{3}\left(1-\frac{4 f^{3}}{4 f^{3}+27 g^{2}}\right)=12^{3} \frac{27 g^{2}}{4 f^{3}+27 g^{2}}
$$

and thus

$$
\kappa=\frac{4 f^{3}}{27 g^{2}}
$$

Notice that with these definitions the map (2.2) implies

$$
\kappa(\rho) \kappa(\tau)=-\frac{4 a^{3}}{27 c^{2}} .
$$

Coming back to (2.2), we can easily solve for $j(\tau)$ and $j(\rho)$ in terms of the complex structures of the K3 (2.1). Introducing ${ }^{4}$

$$
\urcorner=12^{3} a^{3} b d+\left[4 a^{3}+27\left(c^{2}-4 b d\right)\right]^{2},
$$

\footnotetext{
${ }^{3}$ The duality group of the heterotic string on $T^{2}$ is $O(2,2 ; \mathbb{Z})$, which can be seen to be isomorphic to $\left(\mathrm{SL}(2, \mathbb{Z})_{\rho} \times \mathrm{SL}(2, \mathbb{Z})_{\tau}\right) \rtimes\left(\mathbb{Z}_{2} \times \mathbb{Z}_{2}\right)$, where the first $\mathbb{Z}_{2}$ exchanges $\rho$ and $\tau$ (i.e. it is given by T-duality along one of the legs of the $T^{2}$ ) and the second $\mathbb{Z}_{2}$ corresponds to a reflection along one of the directions of the $T^{2}$, which flips the choice of complex structure. We restrict to holomorphic fibrations, and thus ignore this second $\mathbb{Z}_{2}$ factor. See [19] for a review.

${ }^{4}$ We note that the quantity 7 is precisely the same (away from the location of the NS5 branes) as the quantity $q$ defined in [10] once we set $c=0$ in the notation of that paper. We will explain this observation in section 5 .
} 
we have

$$
j(\tau)=-8 \frac{4 a^{3}+27\left(c^{2}-4 b d\right)-\sqrt{7}}{b d}
$$

and

$$
j(\rho)=-8 \frac{4 a^{3}+27\left(c^{2}-4 b d\right)+\sqrt{7}}{b d} .
$$

We have chosen a specific branch of the square root when writing these formulas. Once we fiber the eight dimensional moduli over a complex one-dimensional base, this choice of sign is the one agreeing with the convention in which an NS5 induces a monodromy on $\rho$, leaving $\tau$ invariant. Changing the sign corresponds to exchanging $j(\tau)$ and $j(\rho)$, and is thus related to T-duality along one of the legs of the $T^{2}$ fiber. Accordingly, we have that around single zeroes of 7 we have a monodromy involving the T-duality generator. In other words, if $T$ is not a perfect square (as a function of $s$ ) the heterotic dual will be a T-fold. As we will show, this is precisely the case for a bare $I_{1}$ degeneration, i.e. one where the tadpole is not (locally) saturated. Note that when 7 is a perfect square, the heterotic moduli only have monodromies in the $\mathrm{SL}(2, \mathbb{Z})_{\rho} \times \mathrm{SL}(2, \mathbb{Z})_{\tau}$ subgroup of the T-duality group. We emphasize that these backgrounds with reduced monodromy can still be non-geometric if the monodromies do not admit a classical interpretation globally, see for example [9, 20].

Location of the NS5 branes. In the stable degeneration limit we know that the NS5 branes are located at the zeroes of $b$ and $d[14,15]$ (whether it is $b$ or $d$ that vanishes depends on a tensor branch modulus, encoding the choice of the $E_{8}$ factor associated with the small instanton). This statement is also true away from the stable degeneration limit, as we now review.

Notice first that (2.7) factorizes when $b d=0$ :

$$
\text { ㄱ| }\left.\right|_{b d=0}=\left[4 a^{3}+27 c^{2}\right]^{2} \text {. }
$$

Furthermore, we see from $(2.8)$ and $(2.9)$ that $j(\tau)$ stays finite at these points while $j(\rho)$ diverges. This is the expected behavior for an NS5 brane. Hence, we find that the location of the NS5 brane stays unmodified as we go away from the stable degeneration limit. Notice also that generically $\left.7\right|_{b d=0} \neq 0$, so an isolated NS5 brane does not give rise to non-geometry, as expected from general considerations.

\section{Quantum splitting of the $I_{1}$ degeneration in the $E_{8} \times E_{8}$ heterotic string}

In the following, we turn to the details of the local, heterotic $E_{8} \times E_{8}$ compactification on $I_{1}$ with a locally trivial gauge bundle. Already in this innocent looking case the above described T-fold behavior appears. Following [3, 9], we will study this setup away from the large-volume limit for the fiber torus by fibering adiabatically the eight dimensional heterotic/F-theory duality over a common base, thus obtaining a six dimensional map. 


\subsection{One NS5 on top of the $I_{1}$ degeneration}

We will start by considering the system composed of a small instanton on top of an $I_{1}$ degeneration of the K3. For simplicity, we locate the $I_{1}$-NS5 system at $s=0$. Due to the modified Bianchi identity there is no monodromy for $\rho$ around $s=0$, while there is a $\tau \rightarrow \tau+1$ monodromy. We expect this system to admit a geometric description even after quantum corrections, i.e. it should be possible to write an F-theory background whose heterotic dual has the expected monodromy around $s=0$. This is indeed the case, using for instance the Shioda-Inose construction described in [9]. Explicitly, we write

$$
y^{2}=x^{3}+f_{\rho} x z^{4}+g_{\rho} z^{6}
$$

for the $\rho$-fibration, and analogously with $\rho \rightarrow \tau$ for the $\tau$-fibration. The F-theory dual for such a system is then given by

$$
a=-3 f_{\tau} f_{\rho}, \quad b=b_{\tau} b_{\rho}, \quad c=-\frac{27}{2} g_{\tau} g_{\rho}, \quad d=d_{\tau} d_{\rho}
$$

with

$$
\Delta_{\tau}=4 b_{\tau} d_{\tau}, \quad \Delta_{\rho}=4 b_{\rho} d_{\rho} .
$$

Whenever $f_{\tau}, g_{\tau}, f_{\rho}, g_{\rho}$ exist as holomorphic sections of line bundles of the base (as opposed to the generic branched form which follows from (2.8) and (2.9)) one has that 7 is a perfect square globally

$$
\urcorner=3^{12}\left[f_{\tau}^{3} g_{\rho}^{2}-f_{\rho}^{3} g_{\tau}^{2}\right]^{2}
$$

Thus, there is no T-duality monodromy around any point in the base. Equivalently, the condition on the coefficients of the F-theory fibration for having no T-duality monodromy on the heterotic side is that the map (3.2) holds everywhere in the base for globally well defined, holomorphic sections $f_{\tau}, g_{\tau}, f_{\rho}, g_{\rho}$. This is clearly not the case for $a, b, c, d$ generic sections which will not factor globally on the base.

Coming back to the $I_{1}$-NS5 system, we take the ansatz $f_{\rho}, g_{\rho}$ constant and $f_{\tau}=-3$, $g_{\tau}=2+s$. This gives rise to $\Delta_{\tau}=27 s(s+4)$. We have the desired $I_{1}$ degeneration of the $\tau$ fibration at $s=0$. There is, in addition, a second $I_{1}$ degeneration at $s=-4$, which can be included in the analysis without much trouble. Plugging this ansatz into (3.2) we obtain

$$
a=9 f_{\rho}, \quad b=\Delta_{\rho} s(s+4), \quad c=-\frac{27}{2}(2+s) g_{\rho}, \quad d=\frac{27}{16}
$$

where we have arbitrarily chosen to locate both small instantons on the $E_{8}$ factor associated with $b$. By construction, we have that this ansatz keeps $\rho$ constant, while $\tau$ is non-trivially fibered. This corresponds to the case of one NS5 on top of the $I_{1}$ degeneration of the fibration. Note that, as shown in [9], this configuration of globally constant $\rho$ is the only one accessible in the case of compact heterotic K3 compactifications, if we insist on having a geometric interpretation of the heterotic configuration. 


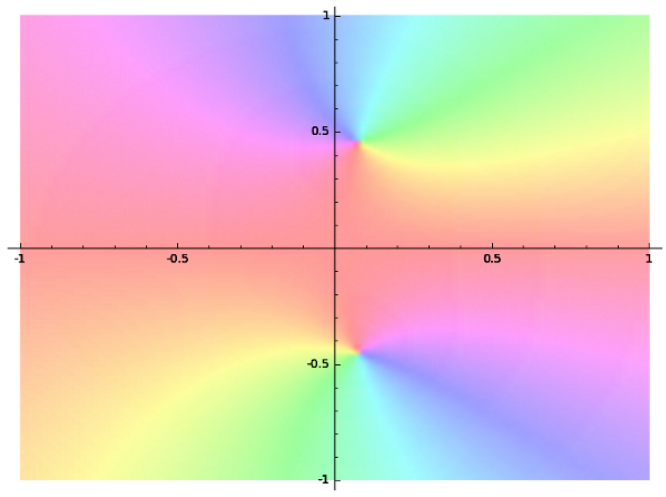

(a) $\epsilon=10^{-1}$.

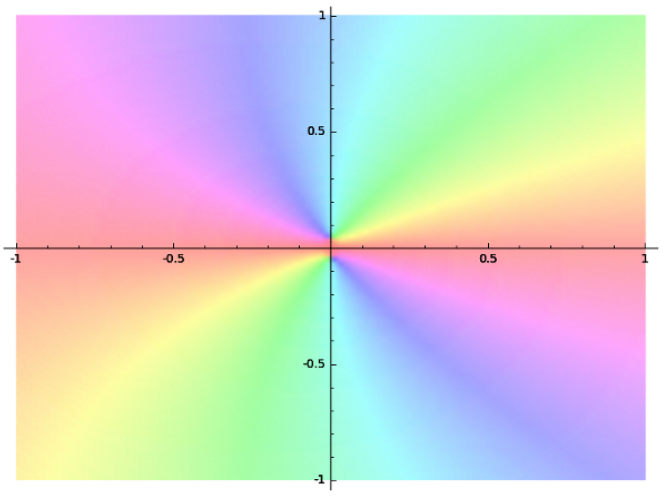

(b) $\epsilon=10^{-3}$.

Figure 1. Plot of 7 close to $s=0$. The hue of the density plot encodes the phase of 7 while the intensity encodes its modulus. For the two plots we took the ansatz (3.6) with $f_{\rho}=-3$, $g_{\rho}=2+\epsilon, \mu=0.5$, and two different values of $\epsilon$. The double zero at $s=0$ in the geometric regime, corresponding to the $I_{1}$ degeneration, splits into two distinct single zeroes. These come together as we take $\epsilon \rightarrow 0$, which corresponds to going to the stable degeneration limit.

\subsection{Moving the NS5 away from the degeneration point}

At this point, a natural question is how to move the NS5 brane away from the $I_{1}$ degeneration. We will answer this question in F-theory, and then dualize back to the heterotic side with the duality map (2.2). We will see that a geometric interpretation is lost, and the $I_{1}$ degeneration splits into its non-geometric components. In order to see this quantum splitting, we just need to choose generic coefficients for the F-theory K3 fibration. For such a generic choice it will no longer be possible to globally factorize the K3 fibration coefficients such that the ansatz (3.2) applies with $f_{\rho}, g_{\rho}, f_{\tau}, g_{\tau}$ holomorphic sections. We can do this in many ways. Let us choose for instance to move the small instanton at $s=0$ to $s=\mu$, while keeping the $a$ and $c$ coefficients unmodified. That is, we choose

$$
a=9 f_{\rho}, \quad b=\Delta_{\rho}(s-\mu)(s+4), \quad c=-\frac{27}{2}(2+s) g_{\rho}, \quad d=\frac{27}{16} .
$$

With this ansatz we have that 7 no longer factorizes and, therefore, we obtain non-trivial branch cuts. The discriminant 7 is in this case a quartic polynomial, so the expression for the roots is rather cumbersome and not very illuminating. Instead of writing it here, we choose to show a plot of 7 in the neighborhood of $s=0$. Figure 1 shows the splitting of the double root as we turn on $\mu$. The splitting of the double root into single roots gives rise to a branch cut in the solutions for $j(\tau)$ and $j(\rho)$, which we display in figure 2 .

Monodromy of the T-fect. The two non-geometric defects, into which the $I_{1}$ singularity splits, are identical up to a choice of duality frame. We denote them (up to duality) by T5. One can easily argue that around a T5 defect one has a monodromy given by $\sigma:(\rho, \tau) \rightarrow(\tau, \rho)$, up to a global $O(2,2 ; \mathbb{Z})$ duality transformation. For example, from (2.7)-(2.8) we notice that the values of $j(\rho)$ and $j(\tau)$ are identical on top of the T5 


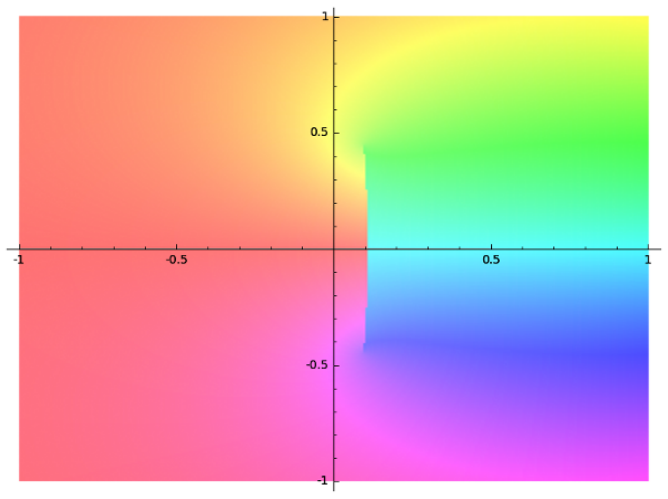

(a) $j(\tau)$.

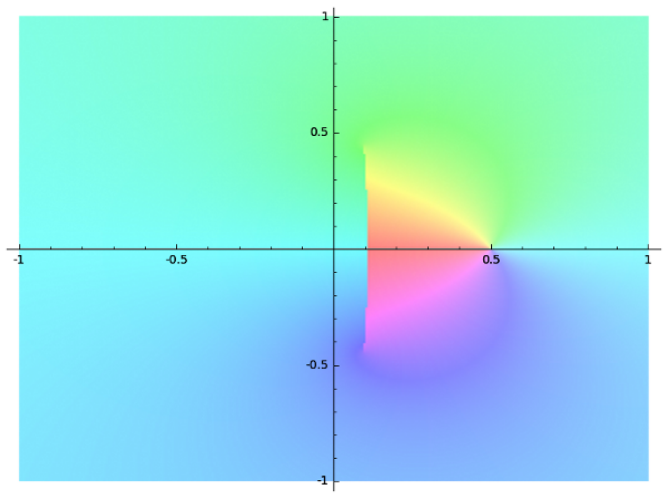

(b) $j(\rho)$.

Figure 2. Values for $j(\tau)$ and $j(\rho)$ for the parameters of figure 1(a). The hue encodes the phase of the $j$-function, while the intensity encodes its modulus. We have labeled the plots according to the classical interpretation of the corresponding sheet when $\epsilon \rightarrow 0$. The branch cut connecting the two components of the $I_{1}$ degeneration exchanges $j(\tau)$ and $j(\rho)$.

and continuously tunable

$$
\left.j(\tau)\right|_{\urcorner=0}=\left.j(\rho)\right|_{\urcorner=0}=-8 \sqrt{-\frac{12^{3} a^{3}}{b d}} .
$$

Therefore, the square of the monodromy, which leaves $j(\tau)$ and $j(\rho)$ invariant, cannot act non-trivially on either $\tau$ and $\rho$, as this would require that $\tau$ and $\rho$ are fixed to values invariant under the corresponding $\mathrm{SL}(2, \mathbb{Z})$ monodromies, and this is a discrete set of possibilities. Alternatively, note that the torus fibers at $7=0$ are generically smooth, and there is no non-trivial $\mathrm{SL}(2, \mathbb{Z})$ monodromy compatible with having a smooth fiber at the origin. This implies that the monodromy action on $(\tau, \rho)$ is of the form

$$
(\tau, \rho) \rightarrow\left(g \rho, g^{-1} \tau\right)
$$

for some $g \in \mathrm{SL}(2, \mathbb{Z})$. But this is simply a conjugation of $\sigma$ by the $O(2,2 ; \mathbb{Z})$ element associated with $g$.

While the local monodromy around a T5 brane is of the form $\sigma$, it must be the case that the monodromy around the two T5 components of the $I_{1}$ geometric degeneration is

$$
\kappa:(\tau, \rho) \rightarrow(\tau+1, \rho-1)
$$

The way this works is depicted schematically in figure 3(a). We have kept the NS5 in the system for simplicity (we will remove it momentarily). Let us denote by $S_{\rho}$ the monodromy associated with the NS5 brane:

$$
S_{\rho}:(\tau, \rho) \rightarrow(\tau, \rho+1)
$$

and similarly $S_{\tau}:(\tau, \rho) \rightarrow(\tau+1, \rho)$. We have chosen the branch cuts in figure 3(a) in a way that makes manifest the local equivalence of the two components of the resolution of the $I_{1}$ 


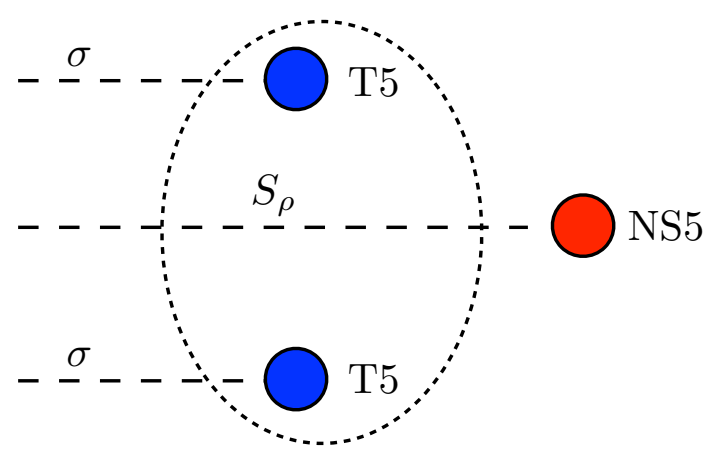

(a) Symmetric presentation.

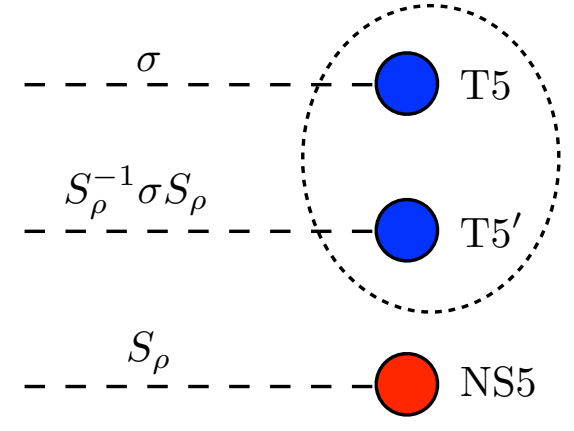

(b) Decoupled presentation.

Figure 3. (a) Quantum behavior of the $I_{1}$ fibration in a heterotic elliptic fibration. The inside of the dotted ellipse becomes in the stable degeneration limit a geometric $I_{1}$ degeneration of the elliptic fiber. We have added a NS5 brane for convenience, and chosen a convenient description for the location of the branch cuts. The monodromy indicated is obtained by crossing the branch cut counter-clockwise. Here $\sigma:(\tau, \rho) \rightarrow(\rho, \tau), S_{\tau}:(\tau, \rho) \rightarrow(\tau+1, \rho)$ and similarly $S_{\rho}:(\tau, \rho) \rightarrow$ $(\tau, \rho+1)$. (b) An alternate presentation, in which the NS5 can be straightforwardly decoupled.

degeneration. It is then clear (by following the dotted contour in figure $3(\mathrm{a})$ ) that in this description the overall monodromy around the $I_{1}$ degeneration is $\kappa=\sigma S_{\rho} \sigma S_{\rho}^{-1}=S_{\tau} S_{\rho}^{-1}$, as expected.

Let us state the result in the absence of NS5s. We can do so by starting with figure 3(a), and pulling the bottom T5 thought the NS5 branch cut. We obtain in this way a T5 brane in a different frame, which in figure $3(\mathrm{~b})$ we denote by $\mathrm{T} 5{ }^{\prime}$. By consistency, the monodromy around the $I_{1}$ degeneration must stay constant, so we must have that the monodromy of the $\mathrm{T} 5^{\prime}$ brane is given by

$$
M_{\mathrm{T} 5^{\prime}}=S_{\rho}^{-1} \sigma S_{\rho}=S_{\tau} S_{\rho}^{-1} \sigma=\kappa \sigma .
$$

Now the NS5 brane can be taken away, leaving us with a consistent description of the $I_{1}$ degeneration.

Let us remark that the above discussion was done in a convenient duality frame, but there is a global frame ambiguity having to do with the $\operatorname{SL}(2, \mathbb{Z})$ frame chosen for $\tau$. Consider for example what happens at an $E_{6}$ degeneration. It splits into $8 I_{1}$ singularities which are not all mutually local. They can for instance be chosen to be of type $(5 \times A, B, 2 \times$ $C)$, in the notation of [21]. Picking a frame in which the monodromy of one chosen $I_{1}$ degeneration is $S_{\tau}$, the monodromy of any other $I_{1}$ degeneration will be $(\tau, \rho) \rightarrow\left(S^{\prime}(\tau), \rho\right)$, with $S^{\prime}=g S_{\tau} g^{-1}$ and $g \in \mathrm{SL}(2, \mathbb{Z})$. This can be achieved in the description above by conjugating every element by the appropriate element $\hat{g} \in O(2,2 ; \mathbb{Z})$, given by the lift of the $\mathrm{SL}(2, \mathbb{Z})$ element $g$.

No dynamics from the T-fect. We have seen that the monodromy around each T5 is of the form $\rho \leftrightarrow \tau$. Thus, on the defect we should have that $\rho=\tau$. It is well known that the heterotic string compactified on a $T^{2}$ develops an enhanced $\mathrm{SU}(2)$ gauge group 
in eight dimensions if the $\tau$ and $\rho$ moduli of the torus are equal. From this, one may erroneously conclude that there is an $\mathrm{SU}(2)$ gauge group on a T5. This would lead to a failure of anomaly cancellation for compact $\mathrm{K} 3 \mathrm{~s}$, and would also contradict the analysis of [22], where it was found that the heterotic string on ADE degenerations with trivial background gauge field did not develop any enhanced symmetry.

The resolution of this puzzle is the following. As pointed out in [3] already, while it is true that on the F-theory dual the K3 fiber on top of the T5 develops an $A_{1}$ singularity, the total space of the fibration of this K3 over $\mathbb{C}$ is smooth, and thus there are no new light degrees of freedom associated with the T5.

We can argue this as follows. The elliptic fibration defined by (2.1) has a discriminant given by

$$
\Delta=u^{10} v^{10}\left(27 d^{2} u^{4}+4 a^{3} u^{2}+54 c d u^{3}+27 c^{2} u^{2}+54 b d u^{2}+54 b c u+27 b^{2}\right) .
$$

The order 10 roots at $u=0$ and $v=0$ come, as expected, from the two $E_{8}$ factors. The resulting discriminant locus $\Delta^{\prime}=\Delta /\left(u^{10} v^{10}\right)$ encodes the position of the remaining four degenerations of the elliptic fiber, each locally of $I_{1}$ type. Thus generically the K3 will be smooth away from the $E_{8}$ points, but we will have extra singularities whenever some of these four remaining roots coincide in the $[u: v]$ plane. This occurs whenever the discriminant of $\Delta^{\prime}$ as a function of $u$ vanishes, i.e.

$$
d^{2} \cdot b^{2} \cdot a^{6} \cdot\left(12^{3} a^{3} b d+\left[4 a^{3}+27\left(c^{2}-4 b d\right)\right]^{2}\right)=0 .
$$

We recognize the last factor as 7 and, thus, we see that $\neg=0$ is associated with extra singularities of the $\mathrm{K} 3$ fiber. This is as expected, since at $j(\rho)=j(\tau)$ the heterotic string on $T^{2}$ has a symmetry enhancement from $E_{8} \times E_{8} \times \mathrm{U}(1)^{2}$ to $E_{8} \times E_{8} \times \mathrm{SU}(2) \times \mathrm{U}(1)$. One can show [16] that the singularity in the K3 is of $I_{2}$ type, which has a local model given by

$$
\sum_{i=1}^{3} z_{i}^{2}=0
$$

in appropriate local variables (given by linear functions of $(x, y, u)$ in an affine patch $v=$ $z=1$ ); we omit the explicit expressions for the redefinitions since they are not particularly illuminating.

We now take $a, b, c, d$ to be functions of a complex coordinate $\mathbb{C}$, and impose that $\urcorner$ vanishes to first order in $s$. In terms of the local model (3.14) this fibration structure is manifested as a base-dependent complex deformation of the $A_{1}$ singularity, which in our setup is given by the local form

$$
\sum_{i=1}^{4} z_{i}^{2}=\delta
$$

with $z_{i}$ given by linear redefinitions of $(x, y, u, s)$, and $\delta \neq 0$. We recognize this space as the deformed conifold, which is indeed smooth. So we see that while the K3 fiber is singular above $s=0$, the total space is smooth, as we claimed above. 
Stable degeneration limit. The classical picture of the heterotic string is obtained when the elliptic $T^{2}$ is of infinite size. ${ }^{5}$ In terms of the $j$-functions, we send $j(\rho) \rightarrow \infty$ while keeping $j(\tau)$ generically finite. Equivalently, we want $\kappa(\rho) \rightarrow-1$ keeping $\kappa(\tau)$ generic. ${ }^{6}$ This can be achieved by a scaling limit described in [15]. A convenient parameterization of this scaling limit is to keep $a, c$ constant, while writing $b d=\epsilon b^{\prime} d^{\prime}$ and sending $\epsilon \rightarrow 0$. This limit was studied above in connection with the location of the NS5 branes, and it indeed gives $j(\rho) \rightarrow \infty$ keeping $j(\tau)$ finite. To leading order in $\epsilon$, we have that $\urcorner$ is a perfect square, as in (2.10). Since 7 is a perfect square we have a well defined geometric interpretation for the background, so we can globally apply the ansatz (3.2), and we end up with the factorized form (3.4). Up to an irrelevant rescaling, for an elliptic curve with $j \rightarrow \infty$ one can choose $(f, g)=(-2,3)$. Plugging this into (3.4), we read that in the stable degeneration limit

$$
\urcorner=3^{12} \Delta_{\tau}^{2}
$$

We observe that in the stable degeneration limit all of the double roots of 7 pair up with roots of the $\tau$ fibration. In other words, this supports the idea that one should think of the T-fects as components of the degenerations of the elliptic $\tau$-fibration, that get resolved by quantum effects.

\subsection{Genericity of the splitting}

In the discussion above we have not quite proven that the splitting always happens unless there is an NS5 brane on top of the $I_{1}$. This can be argued in various ways.

First, let us show this directly in a simple example. We choose to study a case without NS5 branes, given by taking

$$
a=a_{0}, \quad b=b_{0}, \quad c=s, \quad d=1
$$

in (2.1). Both $a_{0}$ and $b_{0}$ are arbitrary constants, parameterizing the moduli space. This is the most general form with these degrees in $s$, up to coordinate redefinitions, except that we have chosen to retain $b_{0}$ general. It can be set to one by a coordinate redefinition, but this obscures the behavior in the stable degeneration limit, which in this parameterization is simply $b_{0} \rightarrow 0$, keeping $a_{0}$ finite but arbitrary. ${ }^{7}$ We are interested in the values of $a_{0}, b_{0}$ for which $\urcorner$ has a double root somewhere in the $s$ plane. Computing the discriminant of 7 with respect to $s$ we obtain

$$
\Delta_{s}(ד) \propto b_{0}^{2} \cdot a_{0}^{6} \cdot\left(a_{0}^{3}+27 b_{0}\right)^{2} .
$$

We recognize the $b_{0}=0$ component as the stable degeneration limit, as discussed above, where 7 becomes a perfect square, and we have two $I_{1}$ degenerations at $s= \pm \sqrt{-\frac{4 a_{0}^{3}}{27}}$. (As we will see momentarily, these $I_{1}$ singularities are mutually non-local.)

\footnotetext{
${ }^{5}$ We should also require that the $10 \mathrm{~d}$ heterotic string coupling is small, which dualizes to the requirement that the $\mathbb{P}^{1}$ base of the dual F-theory K3 is small [12]. Since we will be discussing only the complex structure of the K3 we will ignore this requirement in what follows.

${ }^{6}$ Using (2.5) and (2.6), we see that in the limit, and up to rescalings, $\left(f_{\tau}, g_{\tau}\right)=(a, c)[14]$.

${ }^{7}$ In fact $a_{0}$ will parameterize the complex structure of the $T^{2}$ in the infinite volume limit, see footnote 6 . From $(2.6)$ we have that $a_{0}^{3} / s^{2}=27 \kappa(\tau) / 4$.
} 
We also have solutions away from the stable degeneration limit, at $a_{0}=0$ and $a_{0}^{3}=$ $-27 b_{0}$. Note that since $a_{0}$ parameterizes the complex structure of the elliptic curve it is in general arbitrary in the stable degeneration limit, while the previous families of solutions would go to $j(\tau(s))=0$ in this limit, so that everywhere $\tau=\exp (2 \pi i / 3)$ up to $\operatorname{SL}(2, \mathbb{Z})$ conjugation. This shows that generically the $I_{1}$ singularity splits as we go away from the stable degeneration limit.

The special loci with $a_{0}=0$ correspond in the stable degeneration limit to having a Kodaira singularity of type II, since $\operatorname{deg}(f, g, \Delta)=(\infty, 1,2)$. In this case $\tau$ is constant, and there is no obvious reason from the supergravity viewpoint why the singularity should split. And indeed, as we see, it can be taken away from the stable degeneration limit without inducing non-geometry.

A less hands-on argument goes as follows. Assume that there was a way of moving the NS5 away from the $I_{1}$ degeneration without losing the geometric picture (that is, keeping a globally well defined separation between $\tau$ and $\rho$ ). Since the total monodromy of the system in $\rho$ vanishes, and $\rho \rightarrow \rho+1$ around the NS5, we necessarily have a $\rho \rightarrow \rho-1$ monodromy around the $I_{1}$ degeneration. Supersymmetry requires the fibration to be holomorphic on the base coordinates, so the putative behavior of the elliptic fiber around the $I_{1}$ degeneration should belong to the Kodaira classification. But there is no degeneration with that monodromy, so we conclude that no holomorphic fibration with the right properties exist.

We can also consider what happens when we compactify the base to a $\mathbb{P}^{1}$, so we end up with a compact $\mathrm{K} 3$ on the heterotic side and an elliptically fibered threefold with base a Hirzebruch surface on the F-theory side. As discussed in [9], imposing that the heterotic side has a globally well defined geometric structure fixes rather dramatically the choice of K3s: one finds that $f_{\rho}$ and $g_{\rho}$ should be holomorphic sections of the trivial bundle over the base $\mathbb{P}^{1}$, which forces them to be constants. In other words, the $B$ field is constant, which implies that we have local cancellation of the Bianchi identity, and one NS5 brane on top of every $I_{1}$ degeneration. This is in perfect agreement with the local behavior we have discussed.

\section{F-theory interpretation of the $\mathrm{SO}(32)$ splitting}

We now want to discuss the analogous splitting behavior in the context of the $\mathrm{SO}(32)$ heterotic string. As we will see, from the heterotic viewpoint the conclusions will be identical to those we found in the $E_{8} \times E_{8}$ case. Compactifications of the $\mathrm{SO}(32)$ heterotic string preserving the full $\mathrm{SO}(32)$ gauge group dualize to F-theory backgrounds with simple IIB weak coupling limits. Therefore, our main interest in this regime will be to understand the relation between the splitting we found and the well known splitting of the $\mathrm{O}^{-}$plane in IIB. As we will see, both effects are related.

We will need two ingredients:

1. A map from the $\mathrm{SO}(32)$ string to F-theory away from the stable degeneration limit. We want in particular to have some way of determining $j(\tau), j(\rho)$ for the heterotic $T^{2}$ in terms of the $\mathrm{K} 3$ data, analogous to the expressions in [16]. 
2. A notion of stable degeneration limit, so we can make statements about quantum effects on a classical background.

The map in point $\mathbf{1}$ is provided in [9], for example. We have that for an $\mathrm{SO}(32)$ heterotic $T^{2}$ compactification described by $f_{\tau}, g_{\tau}$ and $f_{\rho}, g_{\rho}$ we have a dual K3 given by

$$
y^{2}=x^{3}+\left(u^{3} v+a u v^{3}+c v^{4}\right) x^{2} z^{2}+b d v^{8} x z^{4}
$$

with

$$
a=-3 f_{\tau} f_{\rho} ; \quad c=-\frac{27}{2} g_{\tau} g_{\rho} ; \quad b d=\frac{1}{16}\left(4 f_{\tau}^{3}+27 g_{\tau}^{2}\right)\left(4 f_{\rho}^{3}+27 g_{\rho}^{2}\right) .
$$

We have denoted by $[u: v]$ the coordinates on the base $\mathbb{P}^{1}$ of the elliptically fibered K3. This K3 is defined by the hypersurface (4.1) on the ambient toric space

$$
\begin{array}{|l|lllll|}
\hline & u & v & x & y & z \\
\hline \mathbb{C}^{*} & 1 & 1 & 4 & 6 & 0 \\
\mathbb{C}^{*} & 0 & 0 & 2 & 3 & 1 \\
\hline
\end{array}
$$

We can now easily check that with these expressions

$$
\begin{aligned}
& j(\tau) j(\rho)=-12^{6} \frac{a^{3}}{27 b d}, \\
& \lambda(\tau) \lambda(\rho)=12^{6} \frac{c^{2}}{4 b d},
\end{aligned}
$$

which are identical to (2.2). Like in the case of the $E_{8} \times E_{8}$ heterotic string, (4.4) is the duality map between the $\mathrm{SO}(32)$ heterotic string on $T^{2}$ and F-theory on the elliptic K3 (4.1) that we will actually use to obtain a six dimensional duality via an adiabatic fibration over a common base. In order to do this we fiber the K3 hypersurface (4.1) over a complex base. Let us study the compact case first. We can obtain it from fibering the K3 in (4.1) over a $\mathbb{P}^{1}$ which we parameterize by $[s: t]$. The most general toric ambient space in this setup is given by (up to redefinitions)

$$
\begin{array}{|l|lllllll|}
\hline & s & t & u & v & x & y & z \\
\hline \mathbb{C}_{1}^{*} & 1 & 1 & 0 & \alpha & \beta & \gamma & 0 \\
\mathbb{C}_{2}^{*} & 0 & 0 & 1 & 1 & 4 & 6 & 0 \\
\mathbb{C}_{3}^{*} & 0 & 0 & 0 & 0 & 2 & 3 & 1 \\
\hline
\end{array}
$$

Homogeneity of (4.1) requires $3 \beta=2 \gamma$, and $\alpha=\beta$. Demanding that the hypersurface is a Calabi-Yau manifold enforces

$$
3 \beta=2+\alpha+\beta+\gamma .
$$

From here we can read off that $\alpha=\beta=-4$ and $\gamma=-6$. This tells us that the total space is an elliptic fibration over an $\mathbb{F}_{4}$ base, as expected [14]. Notice that we have assumed that the coefficient of the $u^{3} v x^{2} z^{2}$ term in (4.1) is identically one. This is not necessary, and 
dropping this assumption leads to the "hidden obstructors" of [23]. We will ignore this possibility for simplicity. Similarly we find

$$
\operatorname{deg}(a)=8 ; \quad \operatorname{deg}(c)=12 ; \quad \operatorname{deg}(b d)=24
$$

viewed as homogeneous polynomials in $(s, t)$. Since the form of the duality map is identical in the parameterization we have chosen, we can simply read off the expression for the $\mathrm{SO}(32)\urcorner$ from (2.7). Furthermore, we have that $\operatorname{deg}(ד)=48$, as in the $E_{8} \times E_{8}$ case.

\subsection{The stable degeneration limit}

We want to take the limit $\rho \rightarrow i \infty$, which implies $j(\rho) \rightarrow \infty$. We can do this, as in the $E_{8} \times E_{8}$ case, by sending $a^{3} / b d$ and $c^{2} / b d$ to infinity while keeping $a^{3} / c^{2} \approx \frac{27}{4} \kappa(\tau)$ arbitrary. A simple way to achieve this is to let $b d$ vanish. To leading order (4.1) becomes in this limit

$$
y^{2}=x^{2}(x+Q)
$$

with $Q=\left(u^{3} v+a u v^{3}+c v^{4}\right) z^{2}$. Since the right hand side of (4.8) has a double root at $x=0$, the discriminant of (4.8) with respect to $x$ vanishes identically. On the other hand, going to the standard Weierstraß form by defining $x=x^{\prime}-\frac{1}{3} Q$ we find that $f$ of the elliptic fibration is given by $-\frac{1}{3} Q^{2}$, and it is in particular generically nonvanishing away from $Q=0$. Thus we learn that in the stable degeneration limit the F-theory elliptic fibration has $j\left(\tau_{\text {IIB }}\right) \rightarrow \infty$ almost everywhere. In other words, the stable degeneration limit on the heterotic side gives the weak coupling limit on the F-theory side.

This is not very surprising: the stable degeneration limit suppresses the world-sheet instanton corrections wrapping the heterotic $T^{2}$. Under S-duality these become D1instantons wrapping the type I $T^{2}$, and then two T-dualities on this $T^{2}$ take us to the IIB side, with the instantons now being $\mathrm{D}(-1)$ branes. But the action of these instantons is suppressed only by the axio-dilaton $C_{0}+i / g_{s}$. Hence, we indeed expect such a behavior: stable degeneration mapping to weak coupling.

As in the $E_{8} \times E_{8}$ case, in the stable degeneration limit we have that $f_{\tau}$ and $g_{\tau}$ are, up to rescalings, equal to $a$ and $c$, respectively. This implies that $T$ is proportional to $\Delta_{\tau}^{2}$, i.e. as in the $E_{8} \times E_{8}$ case 7 only exhibits double zeroes in the stable degeneration limit. These double zeroes are coinciding with the positions of the (geometric) complex structure degeneration points of the heterotic $T^{2}$ fiber. Put differently, assuming that there are no small instantons on top of the singularity, the geometric degenerations only split once we include worldsheet instantons.

In order to have a full understanding of the classical picture, we also want to understand how to read off the position of the small instantons in the stable degeneration limit. By an argument entirely analogous to the one given above for $E_{8} \times E_{8}$, we find that these are located where $b d$ vanishes. As a small sanity check, we notice from the degrees (4.7) that in the compact case we do indeed have 24 instantons. These are required to cancel the tadpole in the compact case. 


\subsection{Dualizing the $I_{1}$ degeneration}

After these preliminaries, let us consider the $\mathrm{SO}(32)$ heterotic background with an $I_{1}$ degeneration. We wish to consider its F-theory dual in the stable degeneration limit, and reinterpret the answer in (weakly coupled) IIB language.

According to the discussion above, an $I_{1}$ singularity on the $\mathrm{SO}(32)$ heterotic side can be described by the dual K3 fibration:

$$
y^{2}=x^{3}+\left(u^{3} v-3 u v^{3}+2(s+1) v^{4}\right) x^{2} z^{2}+\epsilon^{2} v^{8} x z^{4} .
$$

We have chosen to locate the $I_{1}$ degeneration at $s=0$ (there is another $I_{1}$ singularity at $s=-2$ in the stable degeneration limit), gone to the patch $t=1$, and parameterized the stable degeneration limit by $\epsilon \rightarrow 0$. We take $\epsilon^{2}$ instead of $\epsilon$ in (4.9) in order to simplify some later expressions. The discriminant of this elliptic fibration is given by

$$
\Delta_{x}=\epsilon^{4} v^{18}\left(4 v^{6} \epsilon^{2}-\hat{Q}^{2}\right)=\epsilon^{4} v^{18}\left(2 v^{3} \epsilon-\hat{Q}\right)\left(2 v^{3} \epsilon+\hat{Q}\right)
$$

with $\hat{Q}=u^{3}-3 u v^{2}+2(s+1) v^{3}$. We recognize the $v^{18}$ term as the position of the $\mathrm{SO}(32)$ stack: 16 mobile D7 branes on top of an $\mathrm{O}^{-}$. From an eight dimensional perspective, the $\left(2 v^{3} \epsilon \pm \hat{Q}\right)$ terms must be the six components which form the remaining three $\mathrm{O}^{-}$planes in the weak coupling limit. Note that since $u \cdot v$ is in the Stanley Reisner ideal of the toric ambient space (4.5), the polynomials $\hat{Q}=0$ and $v=0$ do not have a common solution. Therefore, $\left(2 v^{3} \epsilon+\hat{Q}\right)$ does not intersect $\left(2 v^{3} \epsilon-\hat{Q}\right)$. In some sense the two branes are parallel to each other. In the weak coupling limit, i.e. $\epsilon \rightarrow 0$, they lie on top of each other and form an $\mathrm{O}^{-}$plane wrapping $\hat{Q}=0 .^{8}$

Let us look more closely at $\hat{Q}=0$ in the vicinity of $s=0$. This cubic in $u$ and $v$ factors at $s=0$ as

$$
\left.\hat{Q}\right|_{s=0}=(u+2 v)(u-v)^{2} .
$$

From this it would seem like we have some exotic physics at $s=0$, coming from the 'coincident' $\mathrm{O}^{-}$planes at $u=v$. However this is not the full story in six dimensions, we also have to keep in mind that we are dealing with a non-trivial fibration over the $[s: t]$-base. Therefore, the gradient of $\hat{Q}$ is three dimensional and at $s=0$ it is given by:

$$
\left.d \hat{Q}\right|_{s=0}=3(u-v)(u+v) d u-6 v(u-v) d v+v^{2}(u+v) d s .
$$

Since the gradient does not vanish, we conclude that the $\mathrm{O}^{-}$worldvolume is smooth at $s=0$ and we expect no extra degrees of freedom.

Let us now discuss what happens as we go away from the stable degeneration limit. We are interested, in particular, in the behavior of the 7-branes away from the $\mathrm{SO}(32)$ stack. For this purpose we go to the patch $v=1$, and take the discriminant of $\Delta_{x}$ as a function of $u$. We obtain

$$
\Delta_{u}\left(\left.\Delta_{x}\right|_{v=1}\right)=\left(s^{2}-\epsilon^{2}\right) \cdot\left((s+2)^{2}-\epsilon^{2}\right) \cdot \epsilon^{46} .
$$

\footnotetext{
${ }^{8}$ Since the vanishing locus of $\hat{Q}$ intersects the $\mathbb{P}^{1}$ base of the K3 fiber at three points, these are the remaining three $\mathrm{O}^{-}$-planes from the eight dimensional perspective.
} 
We find that away from the stable degeneration limit some of the roots coincide at $s= \pm \epsilon$, and $s=-2 \pm \epsilon$. We expect these to be the quantum components of the $I_{1}$ singularities at $s=0$ and $s=-2$ respectively. For concreteness, we focus on the $s= \pm \epsilon$ points which are associated with the original $I_{1}$ degeneration at $s=0$. One easily checks that at both these points the K3 fiber has a singularity of type $I_{2}$, associated with the expected SU(2) symmetry appearing at the self-duality point of T-duality in the heterotic frame. To study the situation in more detail, we concentrate on $s=\epsilon$ for which the discriminant (4.10) becomes

$$
\begin{aligned}
\left.\Delta_{x}\right|_{s=\epsilon, v=1}= & (u+2) \cdot(u-1)^{2} \cdot \epsilon^{4} \cdot\left(u^{3}-3 u+2+4 \epsilon\right) \\
= & \epsilon^{4} \cdot(u+2) \cdot\left(u+2+\frac{4}{9} \epsilon\right) \cdot(u-1)^{2} . \\
& \cdot\left(u-1+2 i \sqrt{\frac{\epsilon}{3}}-\frac{2}{9} \epsilon\right) \cdot\left(u-1-2 i \sqrt{\frac{\epsilon}{3}}-\frac{2}{9} \epsilon\right)+\mathcal{O}\left(\epsilon^{6}\right) .
\end{aligned}
$$

We can interpret this configuration as the slightly split $B, C$ components of a $\mathrm{O}^{-}$when we go away from the weak coupling limit. At $u=-2 v$ and $u=-2 v-\frac{4}{9} \epsilon$ we have the two components of the "ordinary" O7 $^{-}$from before. The two "coincident" O7s from above have recombined such that two mutually local components of the $\mathrm{O}^{-}$planes are still coincident at $u=v$. The remaining two components moved apart to $u=v \pm 2 i \sqrt{\frac{\epsilon}{3}}-\frac{2}{9} \epsilon$. As in the $E_{8} \times E_{8}$ case one can easily check that this $I_{2}$ singularity in the K3 fiber does not lead to singularities in the threefold, once we take the dependence on $s$ into account.

In the case of the $\mathrm{SO}(32)$ heterotic string, we obtain therefore a direct relation between the exotic splitting into non-geometry and the well known splitting of the $\mathrm{O}^{-}$plane: the dual of the heterotic $I_{1}$ degeneration naïvely has two $\mathrm{O}^{-}$planes coinciding on the $\mathrm{K} 3$ fiber (although as explained the $\mathrm{O}^{-}$worldvolume is actually smooth). As we turn on $g_{s}$ the action of $\mathrm{D}(-1)$ instantons splits these $\mathrm{O}^{-}$planes into components, but there is a remnant of the original behavior in that at two slightly displaced points in the base two mutually local components of the $\mathrm{O}^{-}$planes still overlap. These points are dual to the locations of the T5 branes.

\section{Relation to previous work}

The mechanism that we have discussed in this paper explains and illuminates various points in the literature, as we now discuss.

\subsection{Genus-two formulation of $E_{8} \times E_{7}$ compactifications}

As a first point, it was recently pointed out in [10] that the moduli space of non-geometric $E_{8} \times E_{7}$ heterotic compactifications on K3 agrees with the moduli space of F-theory backgrounds with base $\mathbb{F}_{12}$ with $E_{8} \times E_{7}$ singularities on every $\mathrm{K} 3$ fiber, which are also known to describe geometric K3 compactifications of the $E_{8} \times E_{8}$ heterotic strings with instanton number $(0,24)[14,15]$. It was argued in $[10]$ that this is an effect of going away from the stable degeneration limit used in $[14,15]$. We have seen that this explanation is mostly correct, with the refinement that the system away from the stable degeneration limit can sometimes become geometric for certain high codimension subspaces of moduli space. We 
have shown this statement to be true in the particular case of NS5 branes on top of $I_{1}$ degenerations. That the two sets of observations can be related can be made more precise as follows.

Recall [19] that the T-duality monodromy $\rho \leftrightarrow \tau$ can be embedded into the $O(2,2 ; \mathbb{Z})$ duality group as

$$
g=\left(\begin{array}{llll}
1 & 0 & 0 & 0 \\
0 & 0 & 0 & 1 \\
0 & 0 & 1 & 0 \\
0 & 1 & 0 & 0
\end{array}\right) .
$$

This element has $\operatorname{det}(g)=-1$, and thus it belongs to $O(2,2 ; \mathbb{Z})$ but not $\mathrm{SO}(2,2 ; \mathbb{Z})$. The extension to $O(2,3 ; \mathbb{Z})$, if we assume a trivial action on the Wilson line, is simply

$$
f=\left(\begin{array}{ll}
g & 0 \\
0 & 1
\end{array}\right)
$$

which also has $\operatorname{det}(f)=-1$. This is thus an element of $O(2,3 ; \mathbb{Z})$ not in $\mathrm{SO}(2,3 ; \mathbb{Z})$. Being more precise, the element does not involve parity in the torus, so this is in fact an element of $O^{+}(2,3 ; \mathbb{Z})$ not in $\mathrm{SO}^{+}(2,3 ; \mathbb{Z})$ (here and below we use notation from [10]). Such elements act with a minus sign on the modular form $\chi_{35}(\underline{\tau})$. Thus, T-fects associated with $\rho \leftrightarrow \tau$ exchanges should have $\chi_{35}(\underline{\tau})=0$ at their core, and indeed as we pointed out above \urcorner$\propto q=\chi_{35}^{2}(\underline{\tau}) / \chi_{10}(\underline{\tau})$ for compactifications preserving $E_{8} \times E_{8}$, so the two requirements are compatible.

\subsection{Absence of bare $I_{m}$ singularities in the genus two formalism}

Another obscure point which we understand now (and was in fact the motivation for the present work) is that in the classification of [3] there seemed to be no configuration associated with heterotic ADE degenerations without NS5 branes on top. For instance, the simplest $I_{m-n-0}$ singularity for the genus two fibration was interpreted as $m+n$ NS5 branes on an $I_{\min (m, n)}$ singularity, so one could never entirely remove the NS5 branes. Now we can explain this observation simply as the statement that such degenerations without NS5 branes do not appear in heterotic string compactifications, since they get split into separate non-geometric components.

Furthermore, as we discussed above, we have that the T-fects resulting from geometric singularities splitting are elements of $O^{+}(2,3 ; \mathbb{Z})$ but not of $\mathrm{SO}^{+}(2,3 ; \mathbb{Z})=\operatorname{USp}(4 ; \mathbb{Z})$. The classification of $[24,25]$ only considered monodromies in $\operatorname{USp}(4 ; \mathbb{Z})$, and thus the $\rho \leftrightarrow \tau$ $\mathrm{T}$-fects considered in this paper were not included in [3].

\subsection{Non-geometric unfreezing of moduli spaces}

A third previously confusing point was the observation in [9] that the moduli space of the heterotic K3 compactifications did not seem to allow to move the NS5 branes away from the $I_{1}$ degenerations. As we have seen, the resolution of this puzzle is that the moduli space with a geometric interpretation is a subspace of the true moduli space of the heterotic compactification, which is non-geometric at generic points. 


\subsection{A subtlety at low instanton number}

Finally, we would like to highlight a subtlety that becomes apparent in our analysis, regarding the physics of small instantons at ADE singularities in the heterotic string. We focus in particular on the case of the $E_{8} \times E_{8}$ heterotic string with $k$ NS5 branes probing an $I_{m}$ degeneration. This system (or more precisely, its $\mathbb{C}^{2} / \mathbb{Z}_{m}$ limit) was studied in [26], with the conclusion that for $k<2 m$ one needs to replace $m$ by $\left\lfloor\frac{k}{2}\right\rfloor$ in order to obtain the relevant physics. This result becomes rather puzzling once we embed the $\mathbb{C}^{2} / \mathbb{Z}_{m}$ singularity into a $T^{2}$ fibration, since it breaks invariance under T-duality, which exchanges $m$ with $k-m$, keeping $k$ invariant. (We will discuss what happens when $k<m$ below.) Accordingly, comparison with the results in [26] lead the authors of [3] to give an identification of the physics of the genus two $I_{m-n-0}$ degeneration in terms of $m+n$ NS5 branes on $I_{\min (m, n)}$ singularities, which breaks the manifest T-duality invariance of the underlying genus two formalism.

The resolution of the puzzle has to do again with the quantum splitting of the $I_{m}$ singularities. It is easy to check that for $k<2 m$ the F-theory background chosen in [26] in the stable degeneration limit for representing the physics of $k$ instantons on $\mathbb{C}^{2} / \mathbb{Z}_{m}$ cannot be continued to finite fiber size keeping a zero of order $2 m$ in 7 , which is a necessary condition for having a pointlike $\mathbb{C}^{2} / \mathbb{Z}_{m}$ singularity in the base. In order to illustrate this point, let us consider a small modification of the background in [26], which has the same physical properties but is algebraically easier to deal with:

$$
a=-3 ; \quad b=s^{k}\left(4+s^{m}\right) ; \quad c=2+s^{m} ; \quad d=0,
$$

where we used the notation in (2.1). If we continue to finite fiber size by modifying this to

$$
a=-3 ; \quad b=s^{k}\left(4+s^{m}\right) ; \quad c=2+s^{m} ; \quad d=\epsilon
$$

we obtain

$$
\urcorner=729\left(4+s^{m}\right)\left(4+s^{m}-4 \epsilon s^{k}\right) \underbrace{\left(s^{2 m}-4 \epsilon s^{k+m}-16 \epsilon s^{k}\right)}_{\text {roots near } s=0} .
$$

For $k \geq 2 m$ we expect to have no issue with quantum splittings. In this case 7 has a zero of order $2 m$ at $s=0$ which is compatible with the presence of an $I_{m}$ degeneration. A more detailed computation, using (2.8) and (2.9), shows that the local structure of the heterotic fibration close to $s=0$ is indeed of the expected form ${ }^{9}$

$$
j(\tau) \sim \frac{1}{s^{m}} ; \quad j(\rho) \sim \frac{1}{s^{k-m}},
$$

i.e. $k$ NS5 branes on an $I_{m}$ degeneration. On the other hand, we see that for $k<2 m$ T has a zero of order $k$ at $s=0$. More in detail, one can see that close to $s=0$ we have

$$
j(\tau) \sim \frac{1}{s^{\frac{k}{2}}} ; \quad j(\rho) \sim \frac{1}{s^{\frac{k}{2}}}
$$

as expected for the system of $k$ NS5 branes on an $I_{\frac{k}{2}}$ singularity, which is consistent with the prescription in [26]. For $m \leq k<2 m$ it is thus not correct to claim that there is an $I_{m}$

\footnotetext{
${ }^{9}$ Since we are interested in the behaviour of the $j$-functions for $|s| \ll \epsilon$, we expand (2.8) and (2.9) in terms of $s$ to obtain the right leading terms.
} 
degeneration at the origin. Because for the ansatz (5.4) the singularity splits and leaves us (effectively ${ }^{10}$ ) with an $I_{\left\lfloor\frac{k}{2}\right\rfloor}$ singularity, with $\left\lfloor\frac{k}{2}\right\rfloor<m$, at the origin.

Alternatively, for $k \geq m$ one can choose to use the Shioda-Inose ansatz (3.2) in constructing the system of $k$ NS5 branes on top of the $I_{m}$ singularity for finite $T^{2}$ volume. That is, we take

$$
f_{\rho}=-3 ; \quad g_{\rho}=2+\epsilon t^{k-m} ; \quad f_{\tau}=-3 ; \quad g_{\tau}=2+t^{m}
$$

and

$$
b_{\tau}=\frac{1}{4} \Delta_{\tau} ; \quad b_{\rho}=\frac{1}{4} \Delta_{\rho} ; \quad d_{\tau}=d_{\rho}=1,
$$

which is an arbitrary choice of the instanton embedding. In this setting the physics is manifestly invariant under T-duality, i.e. invariant under the $\rho \leftrightarrow \tau$ exchange, and there is no splitting of the degeneration at the origin. The resulting singularity in F-theory, and accordingly the resulting SCFT agrees for $k \geq 2 m$ with the one obtained from (5.4), and generically disagrees for $k<2 m$. For instance, for $k=m$ we obtain the same SCFT as that of $k$ NS5 branes in flat space, with no gauge group, while the prescription of [26] gives a non-trivial gauge group on the tensor branch.

For $k<m$ we cannot write a Shioda-Inose ansatz (since the monodromy on $\rho$ is negative). In this range it is ill-defined to say in any case that we have $k$ NS5 branes on $I_{m}$, since there will be splitting into non-geometry no matter which ansatz we take. In continuing to finite fiber volume from $\mathbb{C}^{2} / \mathbb{Z}_{m}$ we have a genuine choice: we can either still choose the ansatz (5.4) (i.e. effectively setting $m=\left\lfloor\frac{k}{2}\right\rfloor$ ), or choose keeping the degree of 7 maximal (effectively setting $m=k$ ). The second choice leads again to no algebra, agreeing with the claim in [27].

Notice that this freedom in how to move away from the stable degeneration limit can have rather dramatic effects on the SCFT: in the cases where compactifying the fiber gives a quantum deformation of the singularity this translates into a relevant deformation of the SCFT, no matter how large the $T^{2}$ is, as long as it is finite. And since in this context we are discussing the SCFT field theory at the end of the flow, there can be a sharp discontinuity in what we mean by the SCFT associated with $k$ instantons on $I_{m}$ depending on whether we are at finite volume or strictly infinite volume (i.e. $\mathbb{C}^{2} / \mathbb{Z}_{m}$ ). This makes the use of the heterotic/F-theory duality for determining the physics of $k$ instantons on the $\mathbb{C}^{2} / \mathbb{Z}_{m}$ singularity and $k<2 m$ rather subtle. In this case there are various families of quantum corrected $T^{2}$ fibrations which decompactify to $\mathbb{C}^{2} / \mathbb{Z}_{m}$, but which have rather different physics for any finite value of the fiber size. We summarize the situation in figure 4 .

\section{Discussion}

We have shown that the heterotic string on an elliptically fibered K3 (local or global) with small instantons, dynamically develops a non-geometric structure whenever there are not enough NS5 branes on the geometric degenerations to make the contribution of the

\footnotetext{
${ }^{10}$ One can check that the half-integral part of $\frac{k}{2}$ for $k$ odd has no effect on the gauge algebra and multiplet content on the tensor branch of the SCFT, as obtained from the dual F-theory resolution pattern.
} 


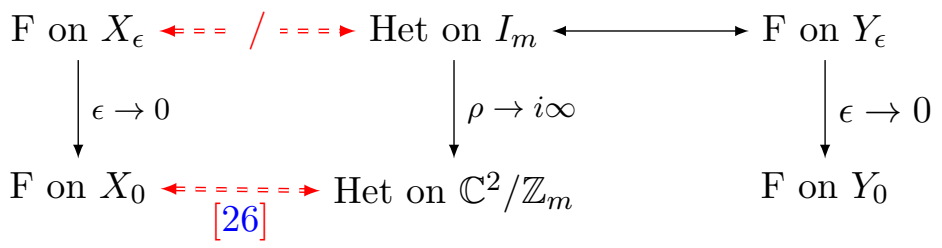

Figure 4. Heterotic/F-theory duality structure for $m \leq k<2 m$. By $X_{\epsilon}$ we denote the family of Calabi-Yau threefolds (5.4), while by $Y_{\epsilon}$ we denote the family (5.8). The struck out dashed arrow on the top row indicates that the heterotic dual of F-theory on $X_{\epsilon}$ does not have an $I_{m}$ singularity at the origin. The dashed line on the bottom row is the duality assumed by [26]. The SCFTs associated to F-theory on $X_{\epsilon}$ and $Y_{\epsilon}$ are generically different.

local system to the modified Bianchi identity everywhere non-negative. ${ }^{11}$ In the case of the compact smooth $\mathrm{K} 3$ there is a very special locus in moduli space where we have exactly one NS5 on top of each $I_{1}$ degeneration. In this case we recover the geometric interpretation, but any deformation away from this point will make the background non-geometric. As we have discussed in section 5 , the existence of this non-geometric splitting resolves a number of puzzles in the literature.

While in this paper we have focused on the simple case of small instantons on K3, it is easy to see that our observation will generalize to a large class of interesting heterotic backgrounds. For instance, we could think about starting from a generic configuration where the NS5 branes are away from the $I_{1}$ degenerations, and going onto the Higgs branch. That is, we "dissolve" the NS5 branes into a smooth bundle. By continuity, we learn that generic heterotic bundles will then also give rise to T-folds, and only over some very specific conditions a standard geometric interpretation will be possible. (As mentioned above, this genericity of non-geometry was already observed in [10] in the case of $E_{8} \times E_{7}$ compactifications on K3 with instanton numbers $(0,24)$, which suffices for making the bundle completely smooth.) Let us emphasize that we are not claiming that every smooth bundle may induce non-geometry, only that this is the generic nature of heterotic compactifications. Specific choices of the bundle might still be geometric. A possible candidate to stay geometric would be the standard embedding, which is known to have the same spectral cover as the system of point-like instantons on $I_{1}$ degenerations [2831], and cancels the Bianchi identity pointwise.

Clearly, non-geometric splitting will also be the generic case for compactifications to fewer than six dimensions, as we can apply the six-dimensional analysis close to neighborhoods of the discriminant locus of the elliptic fibration.

It is well known that existing techniques with high probability give elliptically fibered Calabi-Yaus [32-37], so our observation directly applies to the heterotic string on many of the available backgrounds. It would nevertheless be important to understand if a similar non-geometric splitting holds when there is no elliptic fibration structure. We cannot directly extrapolate our results to this setting, as the heterotic/F-theory duality map is no longer available.

\footnotetext{
${ }^{11}$ As pointed out in section 3.3 there can be exceptions to this statement whenever the local degeneration is compatible with having a constant $\tau$, which is a very non-generic situation.
} 


\section{Acknowledgments}

We thank James Gray, Diego Regalado, Sav Sethi, Timo Weigand and Alberto Zaffaroni for illuminating discussions, and particularly Anamaría Font for collaboration on related earlier work. The work of SM was supported in part by DOE grant DE-SC0009924. This research was supported by the Munich Excellence Cluster for Fundamental Physics "Origin and the Structure of the Universe" and by the ERC Advanced Grant 32004 - Strings and Gravity.

Open Access. This article is distributed under the terms of the Creative Commons Attribution License (CC-BY 4.0), which permits any use, distribution and reproduction in any medium, provided the original author(s) and source are credited.

\section{References}

[1] E. Witten, Small instantons in string theory, Nucl. Phys. B 460 (1996) 541 [hep-th/9511030] [INSPIRE].

[2] D. Lüst, S. Massai and V. Vall Camell, The monodromy of T-folds and T-fects, JHEP 09 (2016) 127 [arXiv: 1508.01193] [InSPIRE].

[3] A. Font, I. García-Etxebarria, D. Lüst, S. Massai and C. Mayrhofer, Heterotic T-fects, $6 D$ SCFTs and F-theory, JHEP 08 (2016) 175 [arXiv: 1603.09361] [INSPIRE].

[4] C.M. Hull, A geometry for non-geometric string backgrounds, JHEP 10 (2005) 065 [hep-th/0406102] [INSPIRE].

[5] A. Sen, F-theory and orientifolds, Nucl. Phys. B 475 (1996) 562 [hep-th/9605150] [INSPIRE].

[6] A. Sen, Orientifold limit of F-theory vacua, Phys. Rev. D 55 (1997) R7345 [hep-th/9702165] [INSPIRE].

[7] T. Banks, M.R. Douglas and N. Seiberg, Probing F-theory with branes, Phys. Lett. B 387 (1996) 278 [hep-th/9605199] [INSPIRE].

[8] F. Denef, Les Houches lectures on constructing string vacua, arXiv:0803.1194 [INSPIRE].

[9] J. McOrist, D.R. Morrison and S. Sethi, Geometries, non-geometries and fluxes, Adv. Theor. Math. Phys. 14 (2010) 1515 [arXiv: 1004 .5447] [InSPIRE].

[10] A. Malmendier and D.R. Morrison, K3 surfaces, modular forms and non-geometric heterotic compactifications, Lett. Math. Phys. 105 (2015) 1085 [arXiv:1406.4873] [INSPIRE].

[11] A. Malmendier and T. Shaska, The Satake sextic in elliptic fibrations on K3, arXiv: 1609.04341 [INSPIRE].

[12] C. Vafa, Evidence for F-theory, Nucl. Phys. B 469 (1996) 403 [hep-th/9602022] [INSPIRE].

[13] J. Gu and H. Jockers, Nongeometric F-theory-heterotic duality, Phys. Rev. D 91 (2015) 086007 [arXiv: 1412.5739] [INSPIRE].

[14] D.R. Morrison and C. Vafa, Compactifications of F-theory on Calabi-Yau threefolds. 1, Nucl. Phys. B 473 (1996) 74 [hep-th/9602114] [INSPIRE].

[15] D.R. Morrison and C. Vafa, Compactifications of F-theory on Calabi-Yau threefolds. 2, Nucl. Phys. B 476 (1996) 437 [hep-th/9603161] [INSPIRE].

[16] G. Lopes Cardoso, G. Curio, D. Lüst and T. Mohaupt, On the duality between the heterotic string and F-theory in eight-dimensions, Phys. Lett. B 389 (1996) 479 [hep-th/9609111] [INSPIRE]. 
[17] W. Lerche and S. Stieberger, Prepotential, mirror map and F-theory on K3, Adv. Theor. Math. Phys. 2 (1998) 1105 [Erratum ibid. 3 (1999) 1199] [hep-th/9804176] [INSPIRE].

[18] S. Tetsuji, Kummer sandwich theorem of certain elliptic K3 surfaces, Proc. Jpn. Acad. Ser. A 82 (2006) 137.

[19] A. Giveon, M. Porrati and E. Rabinovici, Target space duality in string theory, Phys. Rept. 244 (1994) 77 [hep-th/9401139] [INSPIRE].

[20] S. Hellerman, J. McGreevy and B. Williams, Geometric constructions of nongeometric string theories, JHEP 01 (2004) 024 [hep-th/0208174] [INSPIRE].

[21] M.R. Gaberdiel and B. Zwiebach, Exceptional groups from open strings, Nucl. Phys. B 518 (1998) 151 [hep-th/9709013] [INSPIRE].

$[22]$ E. Witten, Heterotic string conformal field theory and A-D-E singularities, JHEP 02 (2000) 025 [hep-th/9909229] [inSPIRE].

[23] P.S. Aspinwall, Point-like instantons and the $\operatorname{Spin}(32) / Z_{2}$ heterotic string, Nucl. Phys. B 496 (1997) 149 [hep-th/9612108] [INSPIRE].

[24] A.P. Ogg, On pencils of curves of genus two, Topology 5 (1966) 355.

[25] Y. Namikawa and K. Ueno, The complete classification of fibres in pencils of curves of genus two, Manuscripta Math. 9 (1973) 143.

[26] P.S. Aspinwall and D.R. Morrison, Point-like instantons on K3 orbifolds, Nucl. Phys. B 503 (1997) 533 [hep-th/9705104] [INSPIRE].

[27] A. Hanany and A. Zaffaroni, Branes and six-dimensional supersymmetric theories, Nucl. Phys. B 529 (1998) 180 [hep-th/9712145] [INSPIRE].

[28] R. Friedman, J.W. Morgan and E. Witten, Vector bundles over elliptic fibrations, alg-geom/9709029 [INSPIRE].

[29] M. Bershadsky, T.M. Chiang, B.R. Greene, A. Johansen and C.I. Lazaroiu, F-theory and linear $\sigma$-models, Nucl. Phys. B 527 (1998) 531 [hep-th/9712023] [INSPIRE].

[30] P.S. Aspinwall and R.Y. Donagi, The heterotic string, the tangent bundle and derived categories, Adv. Theor. Math. Phys. 2 (1998) 1041 [hep-th/9806094] [InSPIRE].

[31] R. Donagi and M. Wijnholt, Gluing branes II: flavour physics and string duality, JHEP 05 (2013) 092 [arXiv: 1112.4854] [INSPIRE].

[32] F. Rohsiepe, Fibration structures in toric Calabi-Yau fourfolds, hep-th/0502138 [INSPIRE].

[33] P. Candelas, A. Constantin and H. Skarke, An abundance of K3 fibrations from polyhedra with interchangeable parts, Commun. Math. Phys. 324 (2013) 937 [arXiv:1207.4792] [INSPIRE].

[34] S.B. Johnson and W. Taylor, Calabi-Yau threefolds with large $h^{2,1}$, JHEP 10 (2014) 023 [arXiv: 1406.0514] [INSPIRE].

[35] J. Gray, A.S. Haupt and A. Lukas, Topological invariants and fibration structure of complete intersection Calabi-Yau four-folds, JHEP 09 (2014) 093 [arXiv: 1405.2073] [INSPIRE].

[36] S.B. Johnson and W. Taylor, Enhanced gauge symmetry in 6D F-theory models and tuned elliptic Calabi-Yau threefolds, Fortsch. Phys. 64 (2016) 581 [arXiv:1605.08052] [InSPIRE].

[37] L.B. Anderson, X. Gao, J. Gray and S.-J. Lee, Multiple fibrations in Calabi-Yau geometry and string dualities, JHEP 10 (2016) 105 [arXiv:1608.07555] [INSPIRE]. 